\title{
Associations of Temperament and Character with Coping Strategies among Office Workers
}

\author{
Hyun Chan Hwang, Sun Mi Kim ${ }^{凶}$, Doug Hyun Han, and Kyoung Joon Min \\ Department of Psychiatry, College of Medicine, Chung-Ang University, Seoul, Republic of Korea
}

\begin{abstract}
Objective Coping can be defined as the reactions to harm or distress individuals take to reduce stress. This study aimed to determine how an individual's temperament and character is correlated with the person's main coping method.

Methods The Korean version of the Ways of Coping Questionnaire and Temperament and Character Inventory-Revised-Short Version were used to evaluate the coping strategies and temperament and character of 1,035 people. Four hierarchical linear regression analyses with the scores of each coping strategy subscale as a dependent variable were performed.

Results High persistence, cooperativeness, self-transcendence, and low harm avoidance were associated with using a problem-focused coping strategy. Being female, high novelty seeking, reward dependence, and self-transcendence were associated with using a supportseeking coping strategy. High novelty seeking, self-transcendence, and low self-directedness were associated with using emotion-focused coping strategy. Being female, high novelty seeking, persistence, cooperativeness, and self-transcendence were associated with using a hopeful-thinking coping strategy.

Conclusion Our results show that there may be specific personality features guiding what kind of coping strategy an individual might use in a given situation.

Psychiatry Investig 2020;17(1):37-46
\end{abstract}

Key Words Personality, Temperament, Character, Coping strategy, Stress.

\section{INTRODUCTION}

Coping can be defined as the reaction to harm or distress individuals take to reduce stress. ${ }^{1}$ Depending on the form of distress and the method of coping, coping may be efficient and able to reduce stress, or it may be unsuccessful and sometimes cause even more stress. ${ }^{2}$ There are many variables that dictate what kind of coping strategy people use. Coping strategies that express emotions were associated with situations that focused on loss, and wishful thinking was associated with situations involving facing a threat. ${ }^{3}$ Vitaliano et al. ${ }^{4}$ reported that men may use more emotion-focused coping strategies, although their findings were not consistent with those of other studies. Older people tend to use more emotion-focused coping strategies,

Received: April 7, 2019 Revised: September 23, 2019

Accepted: September 25, 2019

$\triangle$ Correspondence: Sun Mi Kim, MD, PhD

Department of Psychiatry, Chung-Ang University Hospital, 102 Heukseok-ro, Dongjak-gu, Seoul 06973, Republic of Korea

Tel: +82-2-6299-1519, Fax: +82-2-6298-1508

E-mail: sunmikim706@gmail.com

(c) This is an Open Access article distributed under the terms of the Creative Commons Attribution Non-Commercial License (https://creativecommons.org/licenses/bync/4.0) which permits unrestricted non-commercial use, distribution, and reproduction in any medium, provided the original work is properly cited. while younger adults may use support seeking or problem-focused coping strategies. ${ }^{5}$ Among the various factors related to choosing a coping strategy, personality plays a major role. ${ }^{6-10}$

Personality is, broadly speaking, described as a series of common behavioral, cognitive, and emotional patterns in an individual that stems from biological and environment aspects. ${ }^{11}$ Although there are multiple viewpoints and ways to quantify personality, one widely used model is Cloninger's Psychobiological Model of Personality. ${ }^{12}$ Cloninger proposed that personality consists of two parts; temperament, the biological part that is moderately heritable, and character, the psychological part moderately based on the individual's experience in life. ${ }^{13}$ Temperament can be further divided into four parts. ${ }^{12}$ High novelty seeking (NS) can mean impulsive and exploratory behavior, high harm avoidance (HA) can mean cautious and apprehensive behavior, high reward dependence $(\mathrm{RD})$ can mean sympathetic and sentimental behavior, and high persistence (P) can mean ambitious and overachieving behavior. ${ }^{12}$ Character can be divided into three parts. ${ }^{12}$ High self-directedness (SD) refers to being responsible and self-accepting, high cooperativeness (C) to being empathic and helpful, and high selftranscendence (ST) to being spiritual and idealistic. ${ }^{12}$ 
Personality and coping strategy both play independent and interactive roles in influencing physical and mental health. ${ }^{14}$ Moreover, recent studies have suggested that personal temperament and character could be closely associated with the coping strategy in response to stress ${ }^{15,16}$ and progression of medical diseases. ${ }^{16,17}$ In An et al.'s study, ${ }^{18} \mathrm{NS}$, SD, and cooperativeness were associated with stress level in Korean medical school students. In addition, NS was associated with an avoidance coping strategy. ${ }^{18}$ Briskness, perseverance, sensory sensitivity, emotional reactivity, and endurance were associated with the exacerbation of clinical symptoms in patients with asthma. ${ }^{17}$ Relative to alcoholic patients with short term abstinence, alcoholic patients with long term abstinence showed a higher level of HA and emotion-oriented coping. ${ }^{19}$

Although it is well known that personality is a factor in coping, the extent of that relationship is unclear. ${ }^{14}$ There is evidence that coping strategies are moderately stable in a given situation. ${ }^{20,21}$ There are also studies that show that people might have a tendency to choose coping strategies out of habit. ${ }^{22}$ Whether coping strategies are dynamic, everchanging processes or there is a coping strategy that individuals tend to use regardless of their situation, there is no doubt that personalities have a significant influence on the type of coping strategy one uses. ${ }^{14}$ Unfortunately, the current evidence base for the relationship between personality and coping strategies is based on studies with a relatively small number of participants, and these studies usually focus on very specific groups or situations. Therefore, in order to further elucidate the relationship between personalities and coping strategies, we expanded the number of study participants and examined a large group of office workers in Korea.

\section{Study aims}

This study aimed to reveal how an individual's temperament and character correlates with the person's main coping method. Through this study, we hope to estimate which stressful situations the individual will be more vulnerable to, based on their temperament and character. Furthermore, we hope to make a theoretical foundation for developing an individualized education system to teach more accommodating coping methods to office workers based on their individual temperament and character.

\section{METHODS}

\section{Participants and data collection}

The data were collected using self-report questionnaires. The research participants were office workers in Seoul over 18 years of age that gave written informed consent. We gathered participants from several local government organizations who worked as management, social workers, and in other various jobs. A total of 1,197 people participated in the study and 1,035 survey sheets were returned. Of the 1,035 survey sheets returned, 42 were dismissed due to invalid replies, resulting in 993 survey sheets to be analyzed. The survey had a final response rate of $95.9 \%$.

\section{Variables collected: dependent variable}

The Korean version of the Ways of Coping Questionnaire (K-WCQ) was used to evaluate the participants' coping strategy. The K-WCQ is based on the Ways of Coping Questionnaire, which was developed based on the cognitive-phenomenological theory of stress and coping. ${ }^{23}$ It was revised to better suit the Korean population into a 62 -item questionnaire with responses on a 4-point Likert scale and was also validated. ${ }^{24}$ The scale measures each person's score in four coping strategies. The four coping strategies are problem-focused coping strategy (PRO), support-seeking coping strategy (SUP), emotionfocused coping strategy (EMO), and hopeful-thinking coping strategy (HOP). The average score for each coping strategy subscale was used.

\section{Variables collected: independent variables}

The demographic data of the study population were compiled. The data gathered were age, sex, education, family income, and marital status and they were evaluated as independent variables. Education was measured based on the years of education that the individual had. Family income was measured on a 1 to 7 scale, 1 representing a family income of under 1,000 US dollars per month and 7 representing a family income of over 6,000 US dollars per month.

Job characteristics were also compiled. Job grade was defined as either low or high. Working hours were measured based on the average number of hours worked per week. Duration of work in the current workplace was measured in number of years.

Each participant's temperament and character were evaluated using the Temperament and Character Inventory-Revised-Short version (TCI-RS). The temperament subscales were novelty seeking (NS), harm avoidance (HA), reward dependence $(\mathrm{RD})$, and persistence $(\mathrm{P})$; and the character subscales were self-directedness (SD), cooperativeness (C), and self-transcendence (ST). For this study, we used the t-scores of the TCI-RS.

\section{Statistical analysis}

Before performing the regression analysis, Pearson's correlation and the multicollinearity diagnostic were evaluated. We determined that there is no issue of multicollinearity of the variables included in the regression analysis. To determine the 
influence of temperament and character on coping strategy, we performed four hierarchical linear regression analyses with the scores of each coping strategy subscale, PRO, SUP, EMO, and HOP, as dependent variables. Concerning the four hierarchical linear regression analyses, in model 1 , the association of demographic factors with coping strategy subscale score was tested; job-related factors were added in model 2, testing the association of job-related factors beyond the effects of demographic factors; and lastly, temperament-character factors were added in model 3, testing the associations of temperamentcharacter factors beyond the effects of demographic factors and job-related factors. Statistical significance was set $a$ priori at $\alpha=$ 0.05 (two-sided) to limit type-I error. We conducted all analyses using the Complex Samples module of the PASW statistics software package, version 19 (IBM Corp., Armonk, NY, USA).

\section{Compliance with ethical standards}

The present study protocol was reviewed and approved by the Institutional Review Board of Chung-Ang University (IRB
No. 1041078-201703-HRBM-052-01). All procedures performed in studies involving human participants were in accordance with the ethical standards of the institutional and national research committee and with the 1964 Helsinki declaration and its later amendments or comparable ethical standards. Informed consent was submitted by all subjects at the time of enrollment.

\section{RESULTS}

\section{Participants characteristics}

Table 1 summarizes the descriptive information for participants' coping strategies, demographic factors, job-related factors, and temperament-character factors.

\section{Results of hierarchical linear regression analyses}

The results from the four hierarchical linear regression analyses testing the influence of demographic factors, job-related factors, and temperament and character on coping strategy are

Table 1. Characteristics of the study participants

\begin{tabular}{|c|c|c|c|c|c|}
\hline Variables & Mean & $\mathrm{SD}$ & Minimum & Maximum & $\mathrm{N}(\%)$ \\
\hline \multicolumn{6}{|l|}{ Coping strategies (K-WCQ) } \\
\hline Problem-focused & 32.8 & 8.9 & 5.0 & 63.0 & \\
\hline Support-seeking & 9.3 & 3.0 & 0.0 & 18.0 & \\
\hline Emotion-focused & 28.2 & 7.6 & 1.0 & 69.0 & \\
\hline Hopeful-thinking & 18.8 & 5.4 & 1.0 & 36.0 & \\
\hline \multicolumn{6}{|l|}{ Demographic factors } \\
\hline Years of age & 41.1 & 9.9 & 18.0 & 66.0 & \\
\hline Sex (female) & & & & & $544(54.8)$ \\
\hline Years of education & 15.5 & 1.9 & 6.0 & 20.0 & \\
\hline Family income* & 5.8 & 1.2 & 1.0 & 7.0 & \\
\hline Living with a partner (yes) & & & & & $608(61.2)$ \\
\hline \multicolumn{6}{|l|}{ Job-related factors } \\
\hline Job grade $(\text { high })^{\dagger}$ & & & & & $544(54.8)$ \\
\hline Work hours per week & 46.6 & 12.0 & 20.0 & 98.0 & \\
\hline Duration of current workplace, months & 73.7 & 88.1 & 1.0 & 425.0 & \\
\hline \multicolumn{6}{|l|}{ Temperament-character factors (TCI) } \\
\hline Novelty seeking & 50.6 & 12.1 & 2.5 & 99.4 & \\
\hline Harm avoidance & 52.5 & 13.1 & 0.4 & 97.2 & \\
\hline Reward dependence & 48.3 & 13.1 & 0.1 & 93.3 & \\
\hline Persistence & 44.5 & 12.1 & 0.6 & 99.5 & \\
\hline Self-directedness & 48.2 & 11.1 & 2.0 & 79.0 & \\
\hline Cooperativeness & 45.8 & 12.5 & 0.0 & 81.0 & \\
\hline Self-transcendence & 47.8 & 11.4 & 27.0 & 146.0 & \\
\hline
\end{tabular}

*1 to 7: below 1,000 USD (1,000,000 Korean Won) to above 6,000 USD (6,000,000 Korean Won) per month, ${ }^{\dagger}$ Job grade: High job grade: same or higher grade than a management position. TCI: Temperament and Character Inventory, K-WCQ: Korean version of Ways of Coping Questionnaire 
summarized in Tables 2-5 and Figure 1.

\section{Problem-focused coping strategy}

\section{Demographic factors}

Demographic factors, tested in model 1, explained 3.6\% of the variance in the problem-focused coping strategy (Table 2). Older age $(p=0.003)$ and more years of education $(p=0.007)$ were associated with greater PRO score.

\section{Job-related factors}

Job-related factors, tested in model 2, explained an additional $0.6 \%$ of variance in problem-focused coping strategy beyond the effects of demographic factors. Higher job grade was marginally associated with greater PRO score $(\mathrm{p}=0.050)$ beyond the effects of demographic factors.

Temperament-character factors Temperament-character factors, tested in model 3, explained an additional $29.6 \%$ of variance in problem-focused coping strategy beyond the effects of demographic factors and job-related factors. A higher HA score was associated with a lower PRO score $(p=0.002)$ beyond the effects of demographic factors and job-related factors. A greater $\mathrm{P}$ score $(\mathrm{p}<0.001)$, greater $\mathrm{C}$ score $(\mathrm{p}<0.001)$, and greater ST score $(\mathrm{p}<0.001)$ were associated with a greater PRO score beyond the effects of demographic factors and job-related factors. In addition, in this final model including all demographic factors, job-related factors, and temperament-character factors, none of the demographic factors or job-related factors were independently related to the PRO score. This final model explained $33.9 \%$ of variance in problem-focused coping strategy.

\section{Emotion-focused coping strategy}

\section{Demographic factors}

Demographic factors, tested in model 1, explained 1.9\% of the variance in emotion-focused coping strategy (Table 3 ). Female sex was associated with a greater EMO score $(\mathrm{p}=0.014)$.

\section{Job-related factors}

Job-related factors, tested in model 2, explained an additional $0.2 \%$ of variance in emotion-focused coping strategy beyond the effects of demographic factors. None of the job-related factors were associated with EMO score beyond the effects

Table 2. Hierarchical linear regression results of demographic, job-related, and temperament-character factors with problem-focused coping strategy

\begin{tabular}{|c|c|c|c|c|c|c|c|c|c|c|c|c|}
\hline \multirow{2}{*}{ Independent variables } & \multicolumn{4}{|c|}{ Model 1} & \multicolumn{4}{|c|}{ Model 2} & \multicolumn{4}{|c|}{ Model 3} \\
\hline & $\mathrm{B}$ & Beta & $\mathrm{t}$ & $\mathrm{p}$ & B & Beta & $\mathrm{t}$ & $\mathrm{p}$ & $\mathrm{B}$ & Beta & $\mathrm{t}$ & $\mathrm{p}$ \\
\hline \multicolumn{13}{|l|}{ Demographic factors } \\
\hline Age & 0.133 & 0.144 & $3.007^{\dagger}$ & 0.003 & 0.181 & 0.195 & $0.001^{\dagger}$ & 0.001 & 0.030 & 0.032 & 0.635 & 0.526 \\
\hline Sex (female) & -0.098 & -0.006 & -0.133 & 0.894 & -0.091 & -0.005 & 0.903 & 0.903 & -0.147 & -0.008 & -0.231 & 0.817 \\
\hline Years of education & 0.527 & 0.106 & $2.693^{\dagger}$ & 0.007 & 0.528 & 0.107 & $0.007^{\dagger}$ & 0.007 & 0.126 & 0.025 & 0.757 & 0.449 \\
\hline Family income & 0.412 & 0.055 & 1.228 & 0.220 & 0.531 & 0.070 & 0.120 & 0.120 & 0.011 & 0.001 & 0.039 & 0.969 \\
\hline Living with a partner (yes) & -0.115 & -0.006 & -0.0127 & 0.899 & 0.201 & 0.011 & 0.826 & 0.826 & 0.403 & 0.022 & 0.520 & 0.603 \\
\hline \multicolumn{13}{|l|}{ Job-related factors } \\
\hline Job grade (high) & & & & & 2.011 & 0.112 & 0.050 & 0.050 & 0.648 & 0.036 & 0.751 & 0.453 \\
\hline Work hours per week & & & & & -0.012 & -0.018 & 0.640 & 0.640 & 0.000 & 0.001 & 0.019 & 0.985 \\
\hline Duration of current workplace & & & & & 0.032 & 0.028 & 0.517 & 0.517 & 0.051 & 0.045 & 1.228 & 0.220 \\
\hline \multicolumn{13}{|l|}{ TCI } \\
\hline Novelty seeking & & & & & & & & & 0.024 & 0.034 & 1.014 & 0.311 \\
\hline Harm avoidance & & & & & & & & & -0.080 & -0.120 & $-3.187^{\dagger}$ & 0.002 \\
\hline Reward dependence & & & & & & & & & -0.026 & -0.038 & -0.849 & 0.396 \\
\hline Persistence & & & & & & & & & 0.201 & 0.276 & $6.046^{\ddagger}$ & 0.000 \\
\hline Self-directedness & & & & & & & & & 0.049 & 0.063 & 1.337 & 0.182 \\
\hline Cooperativeness & & & & & & & & & 0.173 & 0.246 & $5.756^{\ddagger}$ & 0.000 \\
\hline Self-transcendence & & & & & & & & & 0.210 & 0.283 & $7.314^{\ddagger}$ & 0.000 \\
\hline Statistics of the model & \multicolumn{4}{|c|}{$\mathrm{F}=4.853^{\ddagger}, \mathrm{R}^{2}=0.036$} & \multicolumn{4}{|c|}{$\begin{array}{c}\mathrm{F}=3.576^{\ddagger}, \mathrm{R}^{2}=0.042, \\
\mathrm{~F} \text { Change }=1.431, \\
\mathrm{R}^{2} \text { Change }=0.006\end{array}$} & \multicolumn{4}{|c|}{$\begin{array}{c}\mathrm{F}=21.781^{\ddagger}, \mathrm{R}^{2}=0.339, \\
\mathrm{~F} \text { Change }=40.821^{\ddagger}, \\
\mathrm{R}^{2} \text { Change }=0.296\end{array}$} \\
\hline
\end{tabular}

${ }^{*} \mathrm{p}<0.05,{ }^{\dagger} \mathrm{p}<0.01,{ }^{\ddagger} \mathrm{p}<0.001$. TCI: Temperament and Character Inventory 
Table 3. Hierarchical linear regression results of demographic, job-related, and temperament-character factors with emotion-focused coping strategy

\begin{tabular}{|c|c|c|c|c|c|c|c|c|c|c|c|c|}
\hline \multirow{2}{*}{ Independent variables } & \multicolumn{4}{|c|}{ Model 1} & \multicolumn{4}{|c|}{ Model 2} & \multicolumn{4}{|c|}{ Model 3} \\
\hline & B & Beta & $\mathrm{t}$ & $\mathrm{p}$ & B & Beta & $\mathrm{t}$ & $\mathrm{p}$ & B & Beta & $\mathrm{t}$ & $\mathrm{p}$ \\
\hline \multicolumn{13}{|l|}{ Demoagraphic factors } \\
\hline Age & -0.020 & -0.025 & -0.525 & 0.600 & -0.040 & -0.049 & -0.826 & 0.409 & -0.026 & 0.047 & -0.556 & 0.579 \\
\hline Sex (female) & 1.605 & 0.104 & $2.466^{\dagger}$ & 0.014 & 1.558 & 0.101 & $2.371^{\dagger}$ & 0.018 & 1.245 & 0.643 & 1.935 & 0.053 \\
\hline Years of education & -0.247 & -0.057 & -1.437 & 0.151 & -0.242 & -0.056 & -1.403 & 0.161 & -0.240 & 0.168 & -1.426 & 0.154 \\
\hline Family income1 & -0.342 & -0.052 & -1.161 & 0.246 & -0.364 & -0.055 & -1.213 & 0.226 & -0.455 & 0.292 & -1.556 & 0.120 \\
\hline Living with a partner (yes) & -0.460 & -0.028 & -0.581 & 0.561 & -0.484 & -0.030 & -0.601 & 0.548 & 0.447 & 0.782 & 0.572 & 0.567 \\
\hline \multicolumn{13}{|l|}{ Job-related factors } \\
\hline Job grade (high) & & & & & -0.128 & -0.008 & -0.143 & 0.887 & 0.193 & -0.869 & 0.223 & 0.824 \\
\hline Work hours per week & & & & & 0.007 & 0.012 & 0.306 & 0.760 & -0.004 & 0.023 & -0.187 & 0.852 \\
\hline Duration of current workplace & & & & & 0.043 & 0.044 & 1.000 & 0.318 & 0.047 & 0.042 & 1.126 & 0.261 \\
\hline \multicolumn{13}{|l|}{ TCI } \\
\hline Novelty seeking & & & & & & & & & 0.131 & 0.024 & $5.375^{\ddagger}$ & 0.000 \\
\hline Harm avoidance & & & & & & & & & 0.012 & 0.025 & 0.485 & 0.628 \\
\hline Reward dependence & & & & & & & & & 0.019 & 0.030 & 0.625 & 0.532 \\
\hline Persistence & & & & & & & & & 0.052 & 0.034 & 1.537 & 0.125 \\
\hline Self-directedness & & & & & & & & & -0.127 & 0.037 & $-3.416^{\dagger}$ & 0.001 \\
\hline Cooperativeness & & & & & & & & & -0.003 & 0.030 & -0.097 & 0.923 \\
\hline Self-transcendence & & & & & & & & & 0.107 & 0.029 & $3.691^{\ddagger}$ & 0.000 \\
\hline Statistics of the model & & $\mathrm{F}=2.471^{*}$ & $\mathrm{R}^{2}=0.019$ & & & $\begin{array}{l}\mathrm{F}=1.692, \\
\mathrm{~F} \text { Chang } \\
\mathrm{R}^{2} \text { Chan }\end{array}$ & $\begin{array}{l}\mathrm{R}^{2}=0.021, \\
\mathrm{e}=0.404, \\
\mathrm{e}=0.002\end{array}$ & & & $\begin{array}{l}=5.433^{\ddagger}, \\
\text { F Chang } \\
\mathrm{R}^{2} \text { Chan }\end{array}$ & $\begin{array}{l}R^{2}=0.113 \\
e=9.529 \ddagger \\
e=0.093\end{array}$ & \\
\hline
\end{tabular}

${ }^{*} \mathrm{p}<0.05,{ }^{\dagger} \mathrm{p}<0.01,{ }^{\ddagger} \mathrm{p}<0.001$. TCI : Temperament and Character Inventory

of demographic factors.

\section{Temperament-character factors}

Temperament-character factors, tested in model 3, explained an additional $9.3 \%$ of variance in emotion-focused coping strategy beyond effects of demographic factors and job-related factors. Greater NS score $(\mathrm{p}<0.001)$ and greater ST score $(\mathrm{p}<$ 0.001 ) were associated with greater EMO score beyond effects of demographic factors and job-related factors. Greater SD score $(\mathrm{p}=0.001)$ was associated with lesser EMO score beyond the effects of demographic factors and job-related factors. In addition, in this final model including all demographic factors and job-related factors, none of the demographic factors or jobrelated factors independently related to EMO score. This final model explained $11.3 \%$ of variance in emotion-focused coping strategy.

\section{Support-seeking coping strategy}

\section{Demographic factors}

Demographic factors, tested in model 1, explained $2.5 \%$ of the variance in support-seeking coping strategy (Table 4). Fe- male sex was associated with a greater SUP score $(\mathrm{p}=0.001)$.

\section{Job-related factors}

Job-related factors, tested in model 2, explained an additional $0.05 \%$ of variance in support-seeking coping strategy beyond effects of demographic factors. None of the job-related factors were associated with SUP score beyond the effects of demographic factors.

\section{Temperament-character factors}

Temperament-character factors, tested in model 3, explained an additional $20.7 \%$ of variance in support-seeking coping strategy beyond the effects of demographic factors and job-related factors. Greater NS score $(\mathrm{p}=0.018)$, greater RD score $(\mathrm{p}<$ $0.001)$, and greater ST score $(\mathrm{p}<0.001)$ were associated with greater SUP score beyond effects of demographic factors and job-related factors. Additionally, in this final model including all demographic factors, job-related factors, and temperamentcharacter factors, female sex was independently related to SUP score $(\mathrm{p}=0.025)$. This final model explained $23.7 \%$ of variance in support-seeking coping strategy. 
Table 4. Hierarchical linear regression results of demographic, job-related, and temperament-character factors with support-seeking coping strategy

\begin{tabular}{|c|c|c|c|c|c|c|c|c|c|c|c|c|}
\hline \multirow{2}{*}{ Independent variables } & \multicolumn{4}{|c|}{ Model 1} & \multicolumn{4}{|c|}{ Model 2} & \multicolumn{4}{|c|}{ Model 3} \\
\hline & $\mathrm{B}$ & Beta & $\mathrm{t}$ & $\mathrm{p}$ & B & Beta & $\mathrm{t}$ & $\mathrm{p}$ & B & Beta & $\mathrm{t}$ & $\mathrm{p}$ \\
\hline \multicolumn{13}{|l|}{ Demographic factors } \\
\hline Age & -0.006 & 0.015 & -0.399 & 0.690 & 0.013 & 0.041 & 0.691 & 0.490 & -0.003 & -0.010 & -0.178 & 0.859 \\
\hline Sex (female) & 0.823 & 0.258 & $3.194^{\dagger}$ & 0.001 & 0.856 & 0.140 & $3.296^{\dagger}$ & 0.001 & 0.533 & 0.087 & $2.252^{*}$ & 0.025 \\
\hline Years of education & 0.070 & 0.068 & 1.026 & 0.305 & 0.067 & 0.039 & 0.989 & 0.323 & -0.037 & -0.021 & -0.592 & 0.554 \\
\hline Family income & -.0420 & 0.117 & -0.361 & 0.718 & -0.004 & -0.002 & -0.037 & 0.971 & -0.190 & -0.073 & -1.761 & 0.079 \\
\hline Living with a partner (yes) & -0.085 & 0.313 & -0.272 & 0.786 & 0.005 & 0.001 & 0.017 & 0.986 & 0.009 & 0.001 & 0.033 & 0.974 \\
\hline \multicolumn{13}{|l|}{ Job-related factors } \\
\hline Job grade (high) & & & & & 0.586 & 0.094 & 1.645 & 0.101 & 0.305 & 0.049 & 0.952 & 0.341 \\
\hline Work hours per week & & & & & 0.001 & 0.004 & 0.097 & 0.923 & 0.005 & 0.021 & 0.592 & 0.554 \\
\hline Duration of current workplace & & & & & -0.006 & -0.015 & -0.341 & 0.733 & 0.002 & 0.005 & 0.137 & 0.891 \\
\hline \multicolumn{13}{|l|}{ TCI } \\
\hline Novelty seeking & & & & & & & & & 0.021 & 0.086 & $2.374^{*}$ & 0.018 \\
\hline Harm avoidance & & & & & & & & & -0.014 & -0.061 & -1.519 & 0.129 \\
\hline Reward dependence & & & & & & & & & 0.084 & 0.364 & $7.517^{\ddagger}$ & 0.000 \\
\hline Persistence & & & & & & & & & -0.006 & -0.023 & -0.472 & 0.637 \\
\hline Self-directedness & & & & & & & & & 0.014 & 0.051 & 1.022 & 0.307 \\
\hline Cooperativeness & & & & & & & & & 0.015 & 0.062 & 1.357 & 0.175 \\
\hline Self-transcendence & & & & & & & & & 0.053 & 0.208 & $4.996^{\ddagger}$ & 0.000 \\
\hline Statistics of the model & \multicolumn{4}{|c|}{$\mathrm{F}=3.266^{\dagger}, \mathrm{R}^{2}=0.025$} & \multicolumn{4}{|c|}{$\begin{array}{c}\mathrm{F}=2.435^{*}, \mathrm{R}^{2}=0.029, \\
\mathrm{~F} \text { Change }=1.049, \\
\mathrm{R}^{2} \text { Change }=0.005\end{array}$} & \multicolumn{4}{|c|}{$\begin{array}{c}\mathrm{F}=13.191^{\ddagger}, \mathrm{R}^{2}=0.237, \\
\mathrm{~F} \text { Change }=24.766^{\ddagger} \\
\mathrm{R}^{2} \text { Change }=0.207\end{array}$} \\
\hline
\end{tabular}

${ }^{*} \mathrm{p}<0.05,{ }^{\dagger} \mathrm{p}<0.01,{ }^{\ddagger} \mathrm{p}<0.001$. TCI: Temperament and Character Inventory

\section{Hopeful-thinking coping strategy}

\section{Demographic factors}

Demographic factors, tested in model 1, explained $2.9 \%$ of the variance in hopeful-thinking coping strategy (Table 5). Female sex was associated with greater HOP score $(\mathrm{p}=0.003)$.

\section{Job-related factors}

Job-related factors, tested in model 2, explained an additional $0.2 \%$ of variance in hopeful-thinking coping strategy beyond the effects of demographic factors. None of the job-related factors were associated with HOP score beyond the effects of demographic factors.

\section{Temperament-character factors}

Temperament-character factors, tested in model 3 , explained an additional $16.2 \%$ of variance in hopeful-thinking coping strategy beyond the effects of demographic factors and jobrelated factors. Greater NS score $(\mathrm{p}=0.040)$, greater $\mathrm{P}$ score $(\mathrm{p}<$ $0.001)$, greater $\mathrm{C}$ score $(\mathrm{p}<0.001)$, and greater ST score $(\mathrm{p}<0.001)$ were associated with greater HOP score beyond the effects of demographic factors and job-related factors. Additionally, in this final model including all demographic factors, job-related factors, and temperament-character, female sex remained independently related to HOP score with a marginal statistical significance $(\mathrm{p}=0.014)$. This final model explained $19.4 \%$ of variance in hopeful-thinking coping strategy.

\section{DISCUSSION}

In summary, high persistence, high cooperativeness, high self-transcendence, as well as low harm avoidance were associated with using a problem-focused coping strategy. Being female, and having high novelty seeking, high reward dependence, and high self-transcendence were associated with using a support-seeking coping strategy. In addition, having high novelty seeking, high self-transcendence, and low self-directedness were associated with using emotion-focused coping strategy. Lastly, being female, and having high novelty seeking, high persistence, cooperativeness, and high self-transcendence were associated with using a hopeful-thinking coping strategy.

People that mostly use PRO may be confronted by the stress itself. $^{14,25}$ They should make steps to remove the stress or diminish its impact. ${ }^{14,25}$ As reported in the current study, the cop- 
Table 5. Hierarchical linear regression results of demographic, job-related, and temperament-character factors with hopeful-thinking coping strategy

\begin{tabular}{|c|c|c|c|c|c|c|c|c|c|c|c|c|}
\hline \multirow{2}{*}{ Independent variables } & \multicolumn{4}{|c|}{ Model 1} & \multicolumn{4}{|c|}{ Model 2} & \multicolumn{4}{|c|}{ Model 3} \\
\hline & $\mathrm{B}$ & Beta & $\mathrm{t}$ & $\mathrm{p}$ & B & Beta & $\mathrm{t}$ & $\mathrm{p}$ & $\mathrm{B}$ & Beta & $\mathrm{t}$ & $\mathrm{p}$ \\
\hline \multicolumn{13}{|l|}{ Demographic factors } \\
\hline Age & 0.040 & 0.071 & 1.471 & 0.142 & 0.046 & 0.081 & 1.364 & 0.173 & 0.004 & 0.008 & 0.136 & 0.892 \\
\hline Sex (female) & 1.350 & 0.124 & $2.945^{\dagger}$ & 0.003 & 1.368 & 0.126 & $2.957^{\dagger}$ & 0.003 & 1.075 & 0.099 & $2.476^{*}$ & 0.014 \\
\hline Years of education & 0.215 & 0.070 & 1.772 & 0.077 & 0.215 & 0.070 & 1.767 & 0.078 & 0.086 & 0.028 & 0.754 & 0.451 \\
\hline Family income & 0.271 & 0.058 & 1.307 & 0.192 & 0.291 & 0.062 & 1.374 & 0.170 & 0.064 & 0.014 & 0.326 & 0.745 \\
\hline Living with a partner (yes) & -0.347 & -0.030 & -0.622 & 0.534 & -0.281 & -0.024 & -0.495 & 0.621 & 0.169 & 0.015 & 0.320 & 0.749 \\
\hline \multicolumn{13}{|l|}{ Job-related factors } \\
\hline Job grade (high) & & & & & 0.468 & 0.042 & 0.738 & 0.461 & 0.188 & 0.017 & 0.321 & 0.748 \\
\hline Work hours per week & & & & & 0.013 & 0.031 & 0.801 & 0.423 & 0.017 & 0.040 & 1.087 & 0.277 \\
\hline Duration of current workplace & & & & & 0.022 & 0.032 & 0.736 & 0.462 & 0.029 & 0.041 & 1.022 & 0.307 \\
\hline \multicolumn{13}{|l|}{ TCI } \\
\hline Novelty seeking & & & & & & & & & 0.034 & 0.077 & $2.055^{*}$ & 0.040 \\
\hline Harm avoidance & & & & & & & & & 0.025 & 0.060 & 1.444 & 0.149 \\
\hline Reward dependence & & & & & & & & & 0.006 & 0.015 & 0.300 & 0.764 \\
\hline Persistence & & & & & & & & & 0.083 & 0.184 & $3.659 \ddagger$ & 0.000 \\
\hline Self-directedness & & & & & & & & & -0.044 & -0.091 & -1.764 & 0.078 \\
\hline Cooperativeness & & & & & & & & & 0.081 & 0.187 & $3.971^{\ddagger}$ & 0.000 \\
\hline Self-transcendence & & & & & & & & & 0.136 & 0.297 & $6.945^{\ddagger}$ & 0.000 \\
\hline Statistics of the model & & $\mathrm{F}=3.863^{\dagger}$ & $\mathrm{R}^{2}=0.029$ & & & $\begin{array}{l}\mathrm{F}=2.594^{\dagger}, \\
\mathrm{F} \text { Chang } \\
\mathrm{R}^{2} \text { Chan }\end{array}$ & $\begin{array}{l}R^{2}=0.031, \\
e=3.863^{\dagger}, \\
g e=0.029\end{array}$ & & & $\begin{array}{l}=10.216^{\ddagger} \\
\text { F Change } \\
\mathrm{R}^{2} \text { Chan }\end{array}$ & $\begin{array}{l}R^{2}=0.194 \\
=18.367^{\ddagger}, \\
g e=0.162\end{array}$ & \\
\hline
\end{tabular}

${ }^{*} \mathrm{p}<0.05,{ }^{\dagger} \mathrm{p}<0.01,{ }^{\ddagger} \mathrm{p}<0.001$. TCI: Temperament and Character Inventory

ing trends of people with PRO are associated with lower HA, as well as higher P and ST scores. The HA score of TCI represents avoidance of risky or stressful situations. ${ }^{12}$ This result is consistent with a past survey of 4,355 Korean individuals that found that lower HA and higher $P$ to be associated with a better stress response. ${ }^{26} \mathrm{PRO}$ is known to be mostly used adaptively in workplace situations and was associated with better job satisfaction and mental well-being in nurses and public servants. ${ }^{27-30}$ This may mean that people with lower HA and higher P might be suitable for working in situations with high stress.

In contrast to PRO, people that mostly use EMO aimed to minimize distress triggered by stressors. ${ }^{14,25}$ For reducing distress or escaping from stressful situation, people utilizing EMO used a wide range of response from self-soothing (relaxation) to the expression of negative emotions (e.g., yelling or crying). ${ }^{14,25}$ The coping methods of EMO are associated with higher NS and lower SD temperament scores. The NS scores of the TCI are known to be associated with the expression of emotion, being quick tempered, and impulsivity. ${ }^{31}$ The SD scores of the TCI were reported to be negatively associated with mood changes in patients with major depressive disorder. ${ }^{32}$ There are multiple studies that show that emotional exhaustion, which is one of the major symptoms of burnout, is related to EMO. ${ }^{33,34}$ There has been debate on whether emotional exhaustion causes people to use EMO or situations or people that use EMO have a high chance of emotional exhaustion. ${ }^{35}$ Devebakan et al. ${ }^{36}$ showed that low SD scores were significantly associated with burnout, and those with low scores often thought of themselves as worthless. Furthermore, Yazici et al. ${ }^{37}$ showed that high NS was positively correlated with burnout. This may mean that people with high NS and low SD scores on the TCI are already a vulnerable group for burnout; the use of EMO might be one of the factors that make them vulnerable to the situation. Further research is needed to determine whether this coping strategy is maladaptive in burnout situations.

People using SUP may handle their situation by asking for help from their friends, family, or colleagues. ${ }^{25,38}$ This type of coping strategy is known to be effective in relieving stresses from the workplace. ${ }^{39,40}$ Our study found high NS and RD to be associated with SUP, with SUP having a stronger correlation with $\mathrm{RD}$. This is to be expected as a previous study showed $\mathrm{RD}$ to be associated with social attachment. ${ }^{13}$ It is noteworthy to mention that although there are many studies that show SUP helps reduce the symptoms of burnout, ${ }^{41-44}$ NS has been posi- 


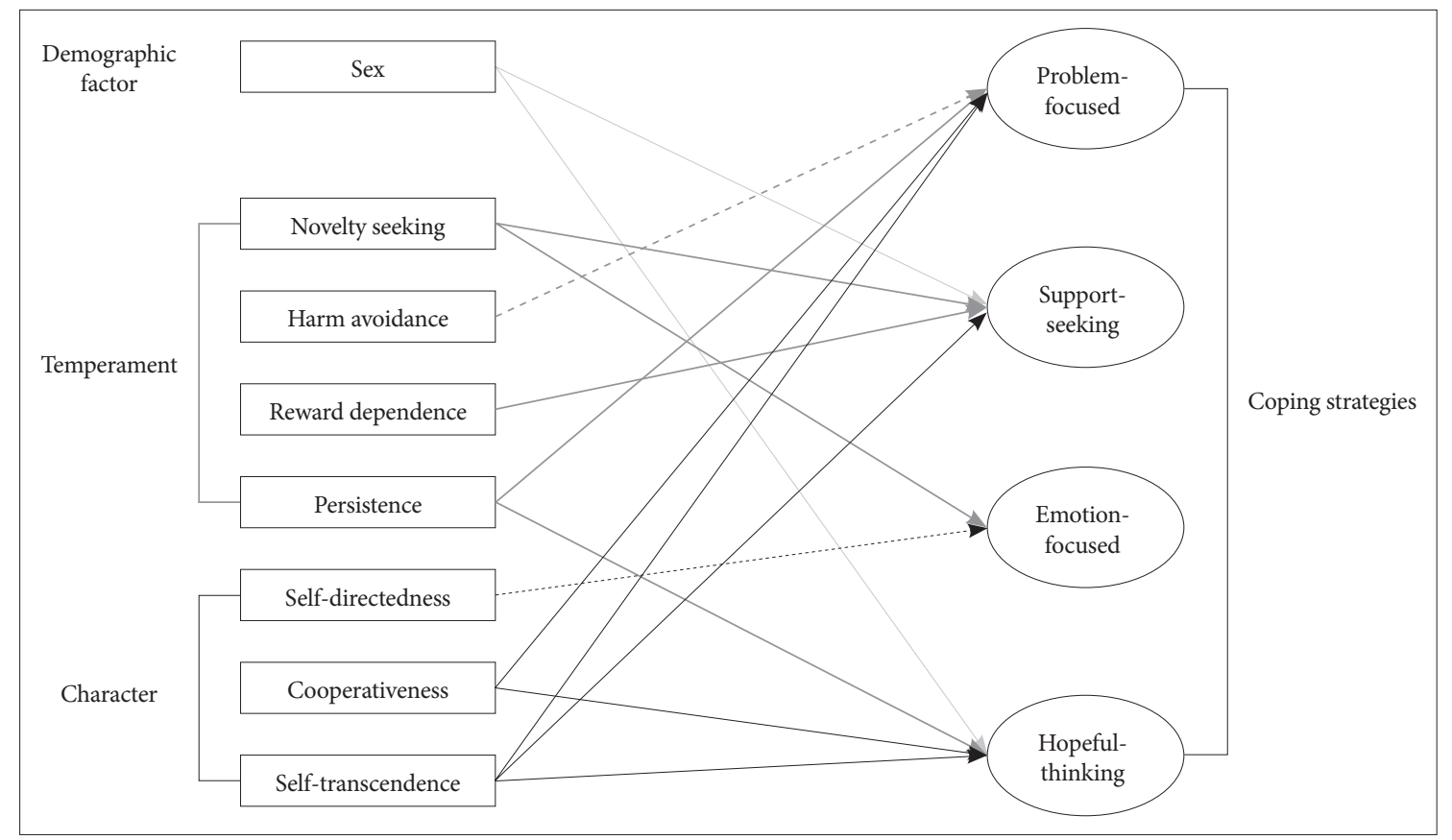

Figure 1. Summary of hierarchical linear regression results. The solid lines indicate positive associations, while the dotted lines indicate negative associations. High persistence $(p<0.001)$, high cooperativeness $(p<0.001)$, high self-transcendence $(p<0.001)$, and low harm avoidance $(p=0.002)$ were associated with using a problem-focused coping strategy. Being female $(p=0.025)$, having high novelty seeking $(p=0.018)$, high reward dependence $(p<0.001)$, and high self-transcendence $(p<0.001)$ were associated with using a support-seeking coping strategy. In addition, having high novelty seeking $(p<0.001)$, high self-transcendence $(p<0.001)$, and low self-directedness $(p=0.001)$ were associated with using emotion-focused coping strategy. Lastly, being female $(p=0.014)$, having high novelty seeking ( $p=0.040)$, high persistence $(p<0.001)$, cooperativeness $(p<0.001)$, and high self-transcendence $(p<0.001)$ were associated with using a hopeful-thinking coping strategy.

tively associated with burnout and $\mathrm{RD}$ has had mixed results. ${ }^{37,45}$

People using HOP focus on managing the negative emotions caused by the problem rather than the problem itself. ${ }^{46}$ Although there is evidence that HOP results in higher levels of negative mood and stress in the work environment, ${ }^{47,48} \mathrm{HOP}$ is not harmful if the situation cannot be changed. ${ }^{49} \mathrm{HOP}$ is said to be maladaptive only when it prevents usage of more adaptive coping strategies. ${ }^{49}$ Our study found that HOP is positively associated with high NS, P, C, and ST. Josefsson et al..$^{50}$ found that high NS, P, and ST are the main factors in predicting changes in personality with age. As personality tends to mature with age, these trait and character groups can be seen as most likely to mature ${ }^{50}$ Furthermore, high $\mathrm{C}$ and $\mathrm{SD}$ is said to show psychological maturity, ${ }^{50}$ although our data failed to show a correlation with high SD. This seems to show similar properties with hope theory, which states that hope is an important factor in change and maturation. ${ }^{51,52}$ To summarize, our data show that individuals with the temperament and character that are most likely to lead to maturation may have a tendency to use coping strategies involving hope, which is also a core factor in maturity. ${ }^{50-52}$

Our study is significant in that it shows the correlation between the trait and characteristics or personality and coping strategies using linear regression in a large number of subjects. Our results show that there may be specific personality features guiding what kind of coping strategy an individual might use in a given situation. There are particular jobs where some coping strategies are more or less preferred than others. . $^{27,30,33,34,39-45,47,48}$ With this information, an individual's personality can be used to guide the person to a more stress-free work environment where they may have a better chance of using adaptive coping strategies than in other situations. Furthermore, some diseases have specific coping strategies that are correlated with a better outcome., 17,20,24,53 Personality information could be used to enhance a doctor's judgment in predicting the patient's outcome.

Our study has a few limitations. First, coping is a complex phenomenon with varying definitions and evaluation methods depending on the researcher. ${ }^{54}$ Our study is based on the works of Folkman and Lazarus, ${ }^{23}$ which may make it difficult to compare our results with other studies based on different coping theories. Second, this is a cross-sectional study. For a better understanding of the relationship between temperament, character, and coping strategies, a longitudinal study may be needed.

In conclusion, our study showed the influence of temperament and character on coping strategies using hierarchical linear regression analyses. Multiple subscales of temperament and character were significantly able to influence the coping strategies, explaining a moderate proportion of the variance. This 
information can be used to guide people to jobs better suited to their personality or coping strategy and predict patient outcomes in specific situations.

\section{Acknowledgments}

\section{None.}

\section{Conflicts of Interest}

The authors have no potential conflicts of interest to disclose.

\section{Author Contributions}

Conceptualization: Sun Mi Kim, Doug Hyun Han. Data curation: Hyun Chan Hwang, Sun Mi Kim. Formal analysis: Sun Mi Kim. Funding acquisition: Doug Hyun Han. Investigation: Sun Mi Kim. Methodology: Hyun Chan Hwang. Project administration: Sun Mi Kim, Doug Hyun Han. Resources: Hyun Chan Hwang, Sun Mi Kim. Supervision: Kyoung Joon Min. Validation: Kyoung Joon Min. Visualization: Hyun Chan Hwang, Sun Mi Kim. Writing_original draft: Hyun Chan Hwang, Sun Mi Kim. Writingreview \& editing: Doug Hyun Han, Kyoung Joon Min.

\section{ORCID iDs}

$\begin{array}{ll}\text { Sun Mi Kim } & \text { https://orcid.org/0000-0003-4131-0542 } \\ \text { Hyun Chan Hwang } & \text { https://orcid.org/0000-0001-6514-5188 } \\ \text { Doug Hyun Han } & \text { https://orcid.org/0000-0001-5888-0686 } \\ \text { Kyoung Joon Min } & \text { https://orcid.org/0000-0003-2866-0273 }\end{array}$

\section{REFERENCES}

1. Abbott J, Dodd M, Gee L, Webb K. Ways of coping with cystic fibrosis: implications for treatment adherence. Disabil Rehabil 2001;23:315-324.

2. Libin E. Coping intelligence: efficient life stress management. Front Psychol 2017;8:302.

3. McCrae RR. Situational determinants of coping responses: loss, threat, and challenge. J Pers Soc Psychol 1984;46:919-928.

4. Vitaliano PP, Russo J, Carr JE, Maiuro RD, Becker J. The ways of coping checklist: revision and psychometric properties. Multivariate Behav Res 1985;20:3-26.

5. Folkman S, Lazarus RS, Pimley S, Novacek J. Age differences in stress and coping processes. Psychol Aging 1987;2:171-184.

6. Parkes KR. Locus of control, cognitive appraisal, and coping in stressful episodes. J Pers Soc Psychol 1984;46:655-668.

7. Parkes KR. Coping in stressful episodes: the role of individual differences, environmental factors, and situational characteristics. J Pers Soc Psychol 1986;51:1277-1292.

8. Vingerhoets AJ, Flohr PJ. Type A behaviour and self-reports of coping preferences. Br J Med Psychol 1984;57(Pt 1):15-21.

9. Costa PT Jr, McCrae RR, Zonderman AB, Barbano HE, Lebowitz B, Larson DM. Cross-sectional studies of personality in a national sample: 2. Stability in neuroticism, extraversion, and openness. Psychol Aging 1986;1:144-149.

10. Scheier MF, Weintraub JK, Carver CS. Coping with stress: divergent strategies of optimists and pessimists. J Pers Soc Psychol 1986;51:12571264.

11. McAdams DP, Olson BD. Personality development: continuity and change over the life course. Annu Rev Psychol 2010;61:517-542.

12. Sung SM, Kim JH, Yang E, Abrams KY, Lyoo IK. Reliability and validity of the Korean version of the Temperament and Character Inventory. Compr Psychiatry 2002;43:235-243.

13. Cloninger CR, Svrakic DM, Przybeck TR. A psychobiological model of temperament and character. Arch Gen Psychiatry 1993;50:975-990.

14. Carver CS, Connor-Smith J. Personality and coping. Annu Rev Psychol 2010;61:679-704.

15. Arango V, Ruggiero DA, Callaway JL, Anwar M, Mann JJ, Reis DJ.
Catecholaminergic neurons in the ventrolateral medulla and nucleus of the solitary tract in the human. J Comp Neurol 1988;273:224-240.

16. Mosley TH Jr, Perrin SG, Neral SM, Dubbert PM, Grothues CA, Pinto BM. Stress, coping, and well-being among third-year medical students. Acad Med 1994;69:765-767.

17. Panek M, Pietras T, Witusik A, Wieteska L, Malachowska B, Mokros L, et al. Identification and association of relationships between selected personal and environmental factors and formal components of temperament and strategies of coping with stress in asthmatic patients. Physiol Behav 2015;149:269-278.

18. An H, Chung S, Park J, Kim SY, Kim KM, Kim KS. Novelty-seeking and avoidant coping strategies are associated with academic stress in Korean medical students. Psychiatry Res 2012;200:464-468.

19. Ando B, Must A, Kurgyis E, Szkaliczki A, Drotos G, Rozsa S, et al. Personality traits and coping compensate for disadvantageous decisionmaking in long-term alcohol abstinence. Alcohol Alcohol 2012;47:18-24.

20. Gil KM, Wilson JJ, Edens JL. The stability of pain coping strategies in young children adolescents, and adults with sickle cell disease over an 18-month period. Clin J Pain 1997;13:110-115.

21. Powers DV, Gallagher-Thompson D, Kraemer HC. Coping and depression in Alzheimer's caregivers: longitudinal evidence of stability. J Gerontol B Psychol Sci Soc Sci 2002;57:P205-211.

22. Moos RH, Holahan CJ. Dispositional and contextual perspectives on coping: toward an integrative framework. J Clin Psychol 2003;59:13871403.

23. Folkman S, Lazarus RS. If it changes it must be a process: study of emotion and coping during three stages of a college examination. J Pers Soc Psychol 1985;48:150-170.

24. Kim JH, Lee JH. Influences of perceived stress, cognitive set, and coping strategies on depression. Korean J Couns Psychother 1988;1:25-45.

25. Folkman S, Lazarus RS, Gruen RJ DeLongis A. Appraisal, coping, health status, and psychological symptoms. J Pers Soc Psychol 1986; 50:571-579.

26. Kim JW, Lee HK, Lee K. Influence of temperament and character on resilience. Compr Psychiatry 2013;54:1105-1110.

27. Boumans NPG, Landeweerd JA. The role of social support and coping behaviour in nursing work: main or buffering effect? Work Stress 1992;6:191-202

28. O'Brien TB, DeLongis A. The interactional context of problem-, emotion-, and relationship-focused coping: the role of the big five personality factors. J Pers 1996;64:775-813.

29. Rick J, Guppy A. Coping strategies and mental health in white collar public sector employees. Eur Work Organ Psychol 1994;4:121-137.

30. Guppy A, Weatherstone L. Coping strategies, dysfunctional attitudes and psychological well-being in white collar public sector employees. Work Stress 1997;11:58-67.

31. Cloninger CR. Psychobiology and treatment of borderline personality disorder. Acta Neuropsychiatr 2002;14:60-65.

32. Spittlehouse JK, Pearson JF, Luty SE, Mulder RT, Carter JD, McKenzie $\mathrm{JM}$, et al. Measures of temperament and character are differentially impacted on by depression severity. J Affect Disord 2010;126:140-146.

33. Shin H, Park YM, Ying JY, Kim B, Noh H, Lee SM. Relationships between coping strategies and burnout symptoms: a meta-analytic approach. Prof Psychol Res Pract 2014;45:44-56.

34. Jenaro C, Flores N, Arias B. Burnout and coping in human service practitioners. Prof Psychol Res Pract 2007;38:80-87.

35. Hamid AARM, Musa SA. The mediating effects of coping strategies on the relationship between secondary traumatic stress and burnout in professional caregivers in the UAE. J Ment Health 2017;26:28-35

36. Devebakan N, Dogan O, Ceylan V, Akin E, Kose S. Relationship between temperament and character dimensions of personality and burnout and management in healthcare organization workers. Psychiatr Clin Psychopharmacol 2018;28:73-79.

37. Yazici AB, Esen O, Yazici E, Esen H, Ince M. The relationship between temperament and character traits and burnout among nurses. J Psy- 
chol Psychother 2014;4:154.

38. Chan IYS, Leung MY, Liang Q. The roles of motivation and coping behaviours in managing stress: Qualitative interview study of Hong Kong expatriate construction professionals in mainland China. Int J Environ Res Public Health 2018;15:561.

39. Varhol P. Identify and manage work-related stress. Electron Des 2000; 48:123-124.

40. Eriksson CB, Bjorck JP, Larson LC, Walling SM, Trice GA, Fawcett J, et al. Social support, organisational support, and religious support in relation to burnout in expatriate humanitarian aid workers. Ment Health Relig Cult 2009;12:671-686.

41. Büssing A, Glaser J. Four-stage process model of the core factors of burnout: The role of work stressors and work-related resources. Work Stress 2000;14:329-346.

42. Wiese L, Rothmann S, Storm K. Coping, stress and burnout in the South African police service in KwaZulu-Natal. SA J Indust Psychol 2003;29:71-80.

43. Koeske GF, Koeske RD. Work load and burnout: can social support and perceived accomplishment help? Soc Work 1989;34:243-248.

44. Halbesleben JR. Sources of social support and burnout: a meta-analytic test of the conservation of resources model. J Appl Psychol 2006;91: 1134-1145.

45. Pejušković $B$, Lečić-Toševski $D$, Priebe $S$, Tošković O. Burnout syndrome among physicians-the role of personality dimensions and coping strategies. Psychiatr Danub 2011;23:389-395.
46. Lim M, Sim M, Chae S, Lee WH, Na J, Kim D. Stress coping strategies and quality of life in patients with schizophrenia. J Korean Neuropsychiatr Assoc 2015;54:181-187.

47. Healy CM, McKay MF. Nursing stress: the effects of coping strategies and job satisfaction in a sample of Australian nurses. J Adv Nurs 2000; 31:681-688.

48. Griffith J, Steptoe A, Cropley M. An investigation of coping strategies associated with job stress in teachers. Br J Educ Psychol 1999;69(Pt 4): 517-531.

49. Lazarus RS. Coping theory and research: past, present, and future. Psychosom Med 1993;55:234-247.

50. Josefsson K, Jokela M, Cloninger CR, Hintsanen M, Salo J, Hintsa T, et al. Maturity and change in personality: developmental trends of temperament and character in adulthood. Dev Psychopathol 2013;25:713727.

51. Snyder CR. Hope theory: Rainbows in the mind. Psychol Inq 2002;13: 249-275.

52. Tarhan S, Bacanlı H, Dombaycı MA, Demir M. Quadruple thinking: hopeful thinking. Procedia Soc Behav Sci 2011;12:568-576.

53. Hwang HC, Kim HR, Han DH, Hong JS, Jeong SH, Shin JH, et al. Influence of major coping strategies on treatment non-adherence and severity of comorbid conditions in hemodialysis patients. J Korean Med Sci 2018;33:e148.

54. Kato T. Frequently used coping scales: a meta-analysis. Stress Health 2015;31:315-323. 